\title{
On various quasi ideals in $b$-semirings
}

\author{
G. Mohanraj ${ }^{1 *}$ and M. Palanikumar ${ }^{2}$
}

\begin{abstract}
We introduce six types of quasi ideals in $b$-semirings. Each quasi ideals generated by single element(set) is established. We characterize various 1-regular(2-regular, regular) by using generalized 1-quasi (generalized 2-quasi, generalized quasi) ideal, 1-quasi (2-quasi, quasi) ideal, weak1(2)-right(left) ideal and weak1(2)-ideal. Examples are provided to strengthen our results.
\end{abstract}

\section{Keywords}

1-quasi ideal, 2-quasi ideal, generalized 1-quasi ideal, generalized 2-quasi ideal.

\section{AMS Subject Classification}

$16 \mathrm{Y} 60$

1,2 Department of Mathematics, Annamalai University, Annamalai Nagar-608002, Tamil Nadu, India.

*Corresponding author: ${ }^{1}$ gmohanraaj@gmail.com; ${ }^{2}$ palanimaths86@gmail.com

Article History: Received 14 October 2019; Accepted 26 December 2019

(C)2020 MJM.

\section{Contents}

1 Introduction $\ldots \ldots \ldots \ldots \ldots \ldots \ldots \ldots \ldots \ldots \ldots \ldots, 20$

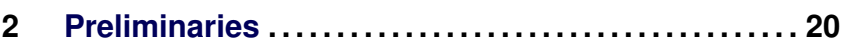

3 1-quasi ideals in b-semirings $\ldots \ldots \ldots \ldots \ldots \ldots \ldots 20$

4 2-quasi ideals in b-semirings $\ldots \ldots \ldots \ldots \ldots \ldots \ldots 23$

References .................................. 26

\section{Introduction}

The concept of $b$-semirings [4] was introduced by Ronnason in 2009. The concept of weak 1(2)-right ideal, weak 1(2)- left ideal, weak 1(2)-ideal in $b$-semirings are introduced by Mohanraj et al [3]. By introducing the 1-regular(2-regular, regular) $b$-semirings. The 1-regular(2-regular, regular) $b$ semirings are characterized by using various weak-ideals by Mohanraj et al [1]. We initiated the notions of $k$-regular $b$ semirings using they are various weak $k$-ideals [2].

\section{Preliminaries}

The algebraic structure $(S,+, \cdot)$ is called a $b$-semiring if $(S,+)$ and $(S, \cdot)$ are semigroups, connected by four distributive laws that "." distributes over " + " from left and right and "+" distributes over "." from left and right[4]. The subset $A$ of $S$ is called a sub $b$-semiring in $S$ if $A$ is itself a $b$-semiring. The subset $A$ of $S$ is called a weak-1 right ideal (weak-1 left ideal) in $S$ if $a_{1}+a_{2} \in A$ and $a_{1} \cdot s \in A\left(s \cdot a_{1} \in A\right)$ for all $a_{1}, a_{2} \in A$ and $s \in S$ [1]. The subset $A$ of $S$ is called a weak-2 right ideal (weak-2 left ideal) in $S$ if $a_{1} \cdot a_{2} \in A$ and $a_{1}+s \in A\left(s+a_{1} \in A\right)$ for all $a_{1}, a_{2} \in A$ and $s \in S$ [1]. The subset $A$ of $S$ is called a weak-1 ideal (weak-2 ideal) in $S$ if it is both weak-1 right ideal (weak-2 right ideal) and weak-1 left ideal(weak-2 left ideal) in $S$ [1]. The subset $A$ of $S$ is called a right ideal (left ideal) in $S$ if it is both weak-1 right ideal (weak-1 left ideal) and weak-2 right ideal(weak-2 left ideal) in $S$ [1]. The $b$-semiring $S$ [1] is called 1-regular [2-regular] if for each $a \in S$ there exists $x \in S$ such that $a \cdot(x \cdot a)=a$ $[a+(x+a)=a]$. The $b$-semiring $S$ [1] is called regular if it is both 1-regular and 2-regular in $S$.

\section{1-quasi ideals in b-semirings}

Throughout this paper, $S$ denotes $b$-semirings unless otherwise noted. The intersection of a weak-1 right ideal and weak-1 left ideal in $S$ is neither weak-1 right ideal nor weak-1 left ideal in $S$ by the following Example 3.1. Naturally one question arises;

What is the intersection of weak 1(2)-right ideal with weak 1(2)-left ideal? We answer the questions by introducing 1-quasi(2-quasi)ideal.

Example 3.1. Consider the b-semiring $S=\left\{g_{1}, g_{2}, g_{3}, g_{4}, g_{5}, g_{6}\right\}$ by the following table.

\begin{tabular}{|l|l|l|l|l|l|l|}
\hline+ & $g_{1}$ & $g_{2}$ & $g_{3}$ & $g_{4}$ & $g_{5}$ & $g_{6}$ \\
\hline$g_{1}$ & $g_{1}$ & $g_{2}$ & $g_{3}$ & $g_{4}$ & $g_{5}$ & $g_{6}$ \\
\hline$g_{2}$ & $g_{1}$ & $g_{2}$ & $g_{3}$ & $g_{4}$ & $g_{5}$ & $g_{6}$ \\
\hline$g_{3}$ & $g_{1}$ & $g_{2}$ & $g_{3}$ & $g_{4}$ & $g_{5}$ & $g_{6}$ \\
\hline$g_{4}$ & $g_{1}$ & $g_{2}$ & $g_{3}$ & $g_{4}$ & $g_{5}$ & $g_{6}$ \\
\hline$g_{5}$ & $g_{1}$ & $g_{2}$ & $g_{3}$ & $g_{4}$ & $g_{5}$ & $g_{6}$ \\
\hline$g_{6}$ & $g_{1}$ & $g_{2}$ & $g_{3}$ & $g_{4}$ & $g_{5}$ & $g_{6}$ \\
\hline
\end{tabular}




\begin{tabular}{|c|c|c|c|c|c|c|}
\hline$\cdot$ & $g_{1}$ & $g_{2}$ & $g_{3}$ & $g_{4}$ & $g_{5}$ & $g_{6}$ \\
\hline$g_{1}$ & $g_{1}$ & $g_{1}$ & $g_{1}$ & $g_{1}$ & $g_{5}$ & $g_{5}$ \\
\hline$g_{2}$ & $g_{1}$ & $g_{2}$ & $g_{1}$ & $g_{4}$ & $g_{5}$ & $g_{6}$ \\
\hline$g_{3}$ & $g_{1}$ & $g_{1}$ & $g_{3}$ & $g_{1}$ & $g_{5}$ & $g_{5}$ \\
\hline$g_{4}$ & $g_{4}$ & $g_{4}$ & $g_{4}$ & $g_{4}$ & $g_{6}$ & $g_{6}$ \\
\hline$g_{5}$ & $g_{1}$ & $g_{1}$ & $g_{5}$ & $g_{1}$ & $g_{5}$ & $g_{5}$ \\
\hline$g_{6}$ & $g_{4}$ & $g_{4}$ & $g_{6}$ & $g_{4}$ & $g_{6}$ & $g_{6}$ \\
\hline
\end{tabular}

Now, $A=\left\{g_{1}, g_{5}\right\}$ and $B=\left\{g_{5}, g_{6}\right\}$ are weak-1 right ideal and weak-1 left ideal respectively, but $A \cap B$ is neither weak-1 right ideal nor weak-1 left ideal in $S$.

Definition 3.2. (i) The subset $Q$ of $S$ is called a generalized 1 -quasi ideal in $S$ if $(Q \cdot S) \cap(S \cdot Q) \subseteq Q$.

(ii) The generalized 1-quasi ideal $Q$ is called a 1-quasi ideal in $S$ if $Q$ is a sub b-semiring.

Lemma 3.3. The generalized 1-quasi ideal $Q$ is a 1-quasi ideal in $S$ if $Q$ is closed under "+".

Proof. Suppose that $Q$ is a generalized 1-quasi ideal which is closed under "+". Now, $Q \cdot Q \subseteq Q \cdot S$ and $Q \cdot Q \subseteq S \cdot Q$ imply $Q \cdot Q \subseteq(Q \cdot S) \cap(S \cdot Q) \subseteq Q$. Thus, $Q$ is a 1 -quasi ideal in $S$.

Remark 3.4. The generalized 1-quasi ideal fails to be a 1quasi ideal in $S$ by the Example 3.5.

Example 3.5. Consider the $b$-semiring

$S=\left\{a_{1}, a_{2}, a_{3}, a_{4}, a_{5}, a_{6}\right\}$ by the following table.

\begin{tabular}{|c|c|c|c|c|c|c|}
\hline+ & $a_{1}$ & $a_{2}$ & $a_{3}$ & $a_{4}$ & $a_{5}$ & $a_{6}$ \\
\hline$a_{1}$ & $a_{1}$ & $a_{1}$ & $a_{1}$ & $a_{1}$ & $a_{5}$ & $a_{5}$ \\
\hline$a_{2}$ & $a_{1}$ & $a_{2}$ & $a_{1}$ & $a_{4}$ & $a_{5}$ & $a_{6}$ \\
\hline$a_{3}$ & $a_{1}$ & $a_{1}$ & $a_{3}$ & $a_{1}$ & $a_{5}$ & $a_{5}$ \\
\hline$a_{4}$ & $a_{4}$ & $a_{4}$ & $a_{4}$ & $a_{4}$ & $a_{6}$ & $a_{6}$ \\
\hline$a_{5}$ & $a_{1}$ & $a_{1}$ & $a_{5}$ & $a_{1}$ & $a_{5}$ & $a_{5}$ \\
\hline$a_{6}$ & $a_{4}$ & $a_{4}$ & $a_{6}$ & $a_{4}$ & $a_{6}$ & $a_{6}$ \\
\hline
\end{tabular}

\begin{tabular}{|c|c|c|c|c|c|c|}
\hline$\cdot$ & $a_{1}$ & $a_{2}$ & $a_{3}$ & $a_{4}$ & $a_{5}$ & $a_{6}$ \\
\hline$a_{1}$ & $a_{1}$ & $a_{1}$ & $a_{1}$ & $a_{1}$ & $a_{1}$ & $a_{1}$ \\
\hline$a_{2}$ & $a_{2}$ & $a_{2}$ & $a_{2}$ & $a_{2}$ & $a_{2}$ & $a_{2}$ \\
\hline$a_{3}$ & $a_{3}$ & $a_{3}$ & $a_{3}$ & $a_{3}$ & $a_{3}$ & $a_{3}$ \\
\hline$a_{4}$ & $a_{4}$ & $a_{4}$ & $a_{4}$ & $a_{4}$ & $a_{4}$ & $a_{4}$ \\
\hline$a_{5}$ & $a_{5}$ & $a_{5}$ & $a_{5}$ & $a_{5}$ & $a_{5}$ & $a_{5}$ \\
\hline$a_{6}$ & $a_{6}$ & $a_{6}$ & $a_{6}$ & $a_{6}$ & $a_{6}$ & $a_{6}$ \\
\hline
\end{tabular}

Clearly, $\left\{a_{2}, a_{5}\right\}$ is a generalized 1-quasi ideal, but $a_{5}+a_{2} \notin$ $\left\{a_{2}, a_{5}\right\}$ implies $\left\{a_{2}, a_{5}\right\}$ is not 1-quasi ideal in $S$.

Lemma 3.6. Every weak-1 right (left) ideal is a 1-quasi ideal in $S$.

Remark 3.7. Converse of the Lemma 3.6 fails by the Example 3.8.

Example 3.8. Consider the $b$-semiring $(S,+, \cdot)$ by the following table.

\begin{tabular}{|c|c|c|c|c|c|c|}
\hline+ & $x_{1}$ & $x_{2}$ & $x_{3}$ & $x_{4}$ & $x_{5}$ & $x_{6}$ \\
\hline$x_{1}$ & $x_{1}$ & $x_{2}$ & $x_{3}$ & $x_{4}$ & $x_{5}$ & $x_{6}$ \\
\hline$x_{2}$ & $x_{1}$ & $x_{2}$ & $x_{3}$ & $x_{4}$ & $x_{5}$ & $x_{6}$ \\
\hline$x_{3}$ & $x_{1}$ & $x_{2}$ & $x_{3}$ & $x_{4}$ & $x_{5}$ & $x_{6}$ \\
\hline$x_{4}$ & $x_{1}$ & $x_{2}$ & $x_{3}$ & $x_{4}$ & $x_{5}$ & $x_{6}$ \\
\hline$x_{5}$ & $x_{1}$ & $x_{2}$ & $x_{3}$ & $x_{4}$ & $x_{5}$ & $x_{6}$ \\
\hline$x_{6}$ & $x_{1}$ & $x_{2}$ & $x_{3}$ & $x_{4}$ & $x_{5}$ & $x_{6}$ \\
\hline
\end{tabular}

\begin{tabular}{|c|c|c|c|c|c|c|}
\hline$\cdot$ & $x_{1}$ & $x_{2}$ & $x_{3}$ & $x_{4}$ & $x_{5}$ & $x_{6}$ \\
\hline$x_{1}$ & $x_{1}$ & $x_{1}$ & $x_{1}$ & $x_{1}$ & $x_{1}$ & $x_{1}$ \\
\hline$x_{2}$ & $x_{1}$ & $x_{2}$ & $x_{2}$ & $x_{4}$ & $x_{4}$ & $x_{4}$ \\
\hline$x_{3}$ & $x_{1}$ & $x_{2}$ & $x_{3}$ & $x_{4}$ & $x_{5}$ & $x_{6}$ \\
\hline$x_{4}$ & $x_{1}$ & $x_{2}$ & $x_{2}$ & $x_{4}$ & $x_{4}$ & $x_{4}$ \\
\hline$x_{5}$ & $x_{1}$ & $x_{2}$ & $x_{3}$ & $x_{4}$ & $x_{5}$ & $x_{6}$ \\
\hline$x_{6}$ & $x_{1}$ & $x_{2}$ & $x_{3}$ & $x_{4}$ & $x_{5}$ & $x_{6}$ \\
\hline
\end{tabular}

Clearly, $\left\{x_{1}, x_{2}\right\}$ is a 1-quasi ideal, but not weak-1 right ideal in $S$.

Theorem 3.9. The intersection of weak-1 right ideal with weak-1 left ideal in $S$ is a 1-quasi ideal.

Proof. For the weak-1 right ideal $A$ and weak-1 left ideal $B$ in $S, A \cap B$ is a sub b-semiring. Now, $[(A \cap B) \cdot S] \cap[S \cdot(A \cap B)] \subseteq$ $(A \cdot S) \cap(S \cdot B) \subseteq A \cap B$ implies $A \cap B$ is a $1-$ quasi ideal in $S$.

Theorem 3.10. For any $a \in S$, the generalized 1-quasi ideal generated by " $a$ ", denoted by $<a>_{g 1 q}$ is given by $\{a\} \cup[(a$. $S) \cap(S \cdot a)]$.

Proof. Now, $x \in(a \cdot S) \cap(S \cdot a)$, then $(x \cdot S) \cap(S \cdot x) \subseteq(a \cdot S) \cap$ $(S \cdot a)$. Thus, $\{a\} \cup[(a \cdot S) \cap(S \cdot a)]$ is a generalized 1-quasi ideal in $S$. If $A$ is a generalized 1-quasi ideal in $S$ such that $a \in A$, then $\{a\} \cup[(a \cdot S) \cap(S \cdot a)] \subseteq A$. Thus $\langle a\rangle_{g 1 q}$ is the generalized 1-quasi ideal generated by " $a$ ".

Corollary 3.11. For a subset $A$ of $S, A \cup[(A \cdot S) \cap(S \cdot A)]$ is the generalized 1-quasi ideal generated by a set $A$ in $S$.

Lemma 3.12. [1] For $n \in \mathbb{Z}^{+}$and $a \in S$,

(i) $(n a \cdot s)=n(a \cdot s)=(a \cdot n s)$.

(ii) $(s \cdot n a)=n(s \cdot a)=(n s \cdot a)$, where $n a=a+a+\ldots n$ times.

(iii) $\left(a^{n}+s\right)=(a+s)^{n}=\left(a+s^{n}\right)$.

(iv) $\left(s+a^{n}\right)=(s+a)^{n}=\left(s^{n}+a\right)$, where $a^{n}=a \cdot a \cdot \ldots n$ times.

Theorem 3.13. For any $a \in S$, the 1 -quasi ideal generated by " $a$ ", denoted by $<a>_{1 q}$ is given by $\left\{\right.$ na $\left.n \in \mathbb{Z}^{+}\right\} \cup[(a \cdot S) \cap$ $(S \cdot a)]$.

Proof. Clearly, $\left\{n a \mid n \in \mathbb{Z}^{+}\right\} \cup[(a \cdot S) \cap(S \cdot a)]$ is generalized 1-quasi ideal. For $x, y \in[(a \cdot S) \cap(S \cdot a)], x+y=a \cdot\left(s_{1}+s_{3}\right) \in$ $a \cdot S$. Similarly, $x+y=\left(s_{2}+s_{4}\right) \cdot a \in S \cdot a$ imply $x+y \in(a \cdot S) \cap$ $(S \cdot a)$. For $x \in\left\{n a \mid n \in \mathbb{Z}^{+}\right\}$and $y \in[(a \cdot S) \cap(S \cdot a)]$ and by Lemma 3.12, $x+y=n a+\left(a \cdot s_{3}\right)=a \cdot\left[(n+1)\left(n a+s_{3}\right)\right] \in a \cdot S$ and $x+y=n a+\left(s_{4} \cdot a\right)=\left[(n+1)\left(n a+s_{4}\right)\right] \cdot a \in S \cdot a$. Thus $x+y \in[(a \cdot S) \cap(S \cdot a)]$. Similarly $y+x \in[(a \cdot S) \cap(S \cdot a)]$. By Lemma 3.3, $\left\{n a \mid n \in \mathbb{Z}^{+}\right\} \cup[(a \cdot S) \cap(S \cdot a)]$ is a 1-quasi ideal in $S$. If $A$ is a 1-quasi ideal in $S$ such that $a \in A$, then $\left\{n a \mid n \in \mathbb{Z}^{+}\right\} \cup[(a \cdot S) \cap(S \cdot a)] \subseteq A$. Hence $\left\langle a>_{1 q}\right.$ is the 1 -quasi ideal generated by " $a$ ". 
Notation 3.14. For a subset $A$ of $S$ and $i=1,2,3, \ldots, n$

(i) $\sum A=\left\{\left(a_{1}+a_{2}+\ldots+a_{n}\right) \mid a_{i} \in A\right\}$.

(ii) $\prod A=\left\{\left(a_{1} \cdot a_{2} \cdot \ldots \cdot a_{n}\right) \mid a_{i} \in A\right\}$.

(iii) $\sum(A \cdot S)=\left\{\left(a_{1} \cdot s_{1}\right)+\left(a_{2} \cdot s_{2}\right)+\ldots+\left(a_{n} \cdot s_{n}\right) \mid a_{i} \in A, s_{i} \in\right.$ S\}.

(iv) $\prod(A+S)=\left\{\left(a_{1}+s_{1}\right) \cdot\left(a_{2}+s_{2}\right) \cdot \ldots \cdot\left(a_{n}+s_{n}\right) \mid a_{i} \in A, s_{i} \in\right.$ $S\}$.

Corollary 3.15. For the subset $A$ of $S, \sum A \cup\left[\sum(A \cdot S) \cap \sum(S\right.$. $A)]$ is the 1-quasi ideal generated by a set $A$ in $S$.

Theorem 3.16. [1] (i) The b-semiring $S$ is 1-regular if and only if

$R \cap L=R \cdot L$, for every weak-1 right ideals $R$ and every weak- 1 left ideals $L$ in $S$.

(ii) The b-semiring $S$ is 2-regular if and only if $R \cap L=R+L$, for every weak-2 right ideals $R$ and every weak-2 left ideals $L$ in $S$.

Theorem 3.17. For a $b$-semiring $S$, the following conditions are equivalent.

(1) $S$ is 1-regular.

(2) $R \cap Q_{1} \subseteq R \cdot Q_{1}$, for the weak-1 right ideals $R$ and generalized 1-quasi ideals $Q_{1}$.

(3) $R \cap Q \subseteq R \cdot Q$, for the weak-1 right ideals $R$ and 1-quasi ideals $Q$.

(4) $Q_{1} \cap L \subseteq Q_{1} \cdot L$, the for generalized 1-quasi ideals $Q_{1}$ and weak-1 left ideals $L$.

(5) $Q \cap L \subseteq Q \cdot L$, for the 1-quasi ideals $Q$ and weak-1 left ideals $L$.

(6) $R \cap L=R \cdot L$, for the weak-1 right ideals $R$ and weak-1 left ideals $L$.

Proof. First, we prove that $(1) \Rightarrow(2) \Rightarrow(3) \Rightarrow(6) \Rightarrow(1)$ and

$(1) \Rightarrow(4) \Rightarrow(5) \Rightarrow(6)$.

(1) $\Rightarrow$ (2) For $a \in R \cap Q_{1}$, then there exampleist $s \in S$ such that $a=(a \cdot s) \cdot a$. Thus, $R \cap Q_{1} \subseteq R \cdot Q_{1}$.

(2) $\Rightarrow$ (3) Straightforward.

(3) $\Rightarrow$ (6) By Lemma 3.6, $R \cap L \subseteq R \cdot L$. Now, $R \cdot L \subseteq R \cdot S \subseteq R$ and $R \cdot L \subseteq S \cdot L \subseteq L$. Then (6) follows.

$(6) \Rightarrow(1)$ The proof follows from Theorem 3.16.

(1) $\Rightarrow(4)$ For $a \in Q_{1} \cap L$, then there exampleist $s \in S$ such that $a=a \cdot(s \cdot a)$. Thus, $Q_{1} \cap L \subseteq Q_{1} \cdot L$.

(4) $\Rightarrow$ (5) Straightforward.

$(5) \Rightarrow(6)$ The proof follows from Lemma 3.6.

Theorem 3.18. For a $b$-semiring $S$, the following conditions are equivalent.

(1) $S$ is 1-regular.

(2) $Q_{1} \cap I \cap Q_{2} \subseteq Q_{1} \cdot I \cdot Q_{2}$, for the generalized 1-quasi ideals $Q_{1}$ and $Q_{2}$ and weak-1 ideals $I$.

(3) $Q_{1} \cap I \cap Q \subseteq Q_{1} \cdot I \cdot Q$, for the generalized 1-quasi ideals $Q_{1}$, weak-1 ideals $I$ and 1 -quasi ideals $Q$.

(4) $Q \cap I \cap Q_{2} \subseteq Q \cdot I \cdot Q_{2}$, for the 1-quasi ideals $Q$, weak-1 ideals I and generalized 1-quasi ideals $Q_{2}$.

(5) $Q \cap I \cap Q \subseteq Q \cdot I \cdot Q$, for the 1-quasi ideals $Q$ and weak-1 ideals $I$

(6) $Q_{1} \cap I \cap L \subseteq Q_{1} \cdot I \cdot L$, for the generalized 1-quasi ideals $Q_{1}$, weak-1 ideals I and weak-1 left ideals $L$.

(7) $Q \cap I \cap L \subseteq Q \cdot I \cdot L$, for the 1 -quasi ideals $Q$, weak-1 ideals
$I$ and weak-1 left ideals $L$.

(8) $R \cap I \cap Q_{2} \subseteq R \cdot I \cdot Q_{2}$, for the weak-1 right ideals $R$, weak-1 ideals $I$ and generalized 1-quasi ideals $Q_{2}$.

(9) $R \cap I \cap Q \subseteq R \cdot I \cdot Q$, for the weak-1 right ideals $R$, weak- 1 ideals I and 1-quasi ideals $Q$.

(10) $R \cap I \cap L \subseteq R \cdot I \cdot L$, for the weak-1 right ideals $R$, weak-1 ideals I and weak-1 left ideals $L$.

(11) $R \cap L=R \cdot L$, for the weak-1 right ideals $R$ and weak-1 left ideals $L$.

(12) $Q_{1} \cap I \subseteq Q_{1} \cdot I \cdot Q_{1}$, for the generalized 1-quasi ideals $Q_{1}$ and weak-1 ideals $I$.

(13) $Q \cap I \subseteq Q \cdot I \cdot Q$, for the 1 -quasi ideals $Q$ and weak-1 ideals $I$.

(14) $Q_{1}=Q_{1} \cdot S \cdot Q_{1}$, for the generalized 1-quasi ideals $Q_{1}$.

(15) $Q=Q \cdot S \cdot Q$, for the 1-quasi ideals $Q$.

Proof. First, we prove that $(1) \Rightarrow(2) \Rightarrow(3) \Rightarrow(5) \Rightarrow(11) \Rightarrow$ (1), (3) $\Rightarrow(6) \Rightarrow(7) \Rightarrow(11),(2) \Rightarrow(4) \Rightarrow(8) \Rightarrow(9) \Rightarrow$ $(10) \Rightarrow(11),(12) \Rightarrow(14) \Rightarrow(1)$ and $(2) \Rightarrow(12) \Rightarrow(13) \Rightarrow$ $(15) \Rightarrow(1)$.

(1) $\Rightarrow$ (2) For $a \in Q_{1} \cap I \cap Q_{2}$, then there exists $s \in S$ such that $a=a \cdot s \cdot a$. Thus, $a=a \cdot(s \cdot a \cdot s) \cdot a \in Q_{1} \cdot I \cdot Q_{2}$. Thus (2) holds.

(2) $\Rightarrow$ (3) Straightforward.

(3) $\Rightarrow$ (5) Straightforward.

(5) $\Rightarrow(11)$ Taking $I=S$ in (5), $R \cap L \subseteq R \cdot L$. Thus, $R \cap L=$ $R \cdot L$.

$(11) \Rightarrow(1)$ The proof follows from Theorem 3.16.

(3) $\Rightarrow(6)$ By Lemma 3.6, (6) holds.

$(6) \Rightarrow(7)$ Straightforward.

(7) $\Rightarrow(11)$ By taking $I=S, R \cap L \subseteq R \cdot L$.Thus, $R \cap L=R \cdot L$.

(2) $\Rightarrow$ (4) Straightforward.

$(4) \Rightarrow(8)$ By Lemma 3.6, we get the result.

$(8) \Rightarrow(9)$ Straightforward.

$(9) \Rightarrow(10)$ The proof follows from Lemma 3.6.

$(10) \Rightarrow(11)$ Taking $I=S$ in (10), $R \cap L \subseteq R \cdot L$. Thus, $R \cap L=$ $R \cdot L$.

$(12) \Rightarrow(14)$ By (12), $Q_{1} \subseteq Q_{1} \cdot S \cdot Q_{1} \subseteq\left[\left(Q_{1} \cdot S\right) \cap\left(S \cdot Q_{1}\right)\right] \subseteq$ $Q_{1}$ implies $Q_{1}=Q_{1} \cdot S \cdot Q_{1}$.

$(14) \Rightarrow$ (1) For any $a \in S, a \in<a\rangle_{g 1 q} \cdot S \cdot\langle a\rangle_{g 1 q}$ and by Theorem 3.10, $a \in[a \cdot S \cdot a] \cup[a \cdot S \cdot[(a \cdot S) \cap(S \cdot a)]] \cup[[(a \cdot$ $S) \cap(S \cdot a)] \cdot S \cdot a] \cup[[(a \cdot S) \cap(S \cdot a)] \cdot S \cdot[(a \cdot S) \cap(S \cdot a)]]$. Thus, $a \in a \cdot S \cdot a$. Therefore $S$ is 1-regular.

$(2) \Rightarrow(12)$ Taking $Q_{2}=Q_{1}$ in (2), we get the result.

(12) $\Rightarrow$ (13) Straightforward.

$(13) \Rightarrow(15)$ By (13), $Q \subseteq Q \cdot S \cdot Q \subseteq[(Q \cdot S) \cap(S \cdot Q)] \subseteq Q$ implies $Q=Q \cdot S \cdot Q$.

$(15) \Rightarrow(1)$ For any $a \in S$ by (15), $a \in<a>_{1 q} \cdot S \cdot\left\langle a>_{1 q}\right.$ and by Theorem 3.13 and Lemma 3.12, $a \in[n a \cdot S \cdot m a] \cup$ $[n a \cdot S \cdot[(a \cdot S) \cap(S \cdot a)]] \cup[[(a \cdot S) \cap(S \cdot a)] \cdot S \cdot m a] \cup[[(a \cdot$ $S) \cap(S \cdot a)] \cdot S \cdot[(a \cdot S) \cap(S \cdot a)]]$. Thus, $a \in a \cdot S \cdot a$. Hence $S$ is 1-regular. 


\section{2-quasi ideals in b-semirings}

Definition 4.1. (i) The subset $Q$ of $S$ is called a generalized 2-quasi ideal in $S$ if $(Q+S) \cap(S+Q) \subseteq Q$.

(ii) The generalized 2-quasi ideal $Q$ is called a 2-quasi ideal in $S$ if $Q$ is a sub b-semiring.

Definition 4.2. The generalized 1-quasi ideal $Q$ is called a generalized quasi ideal if it is generalized 2-quasi ideal.

Definition 4.3. The sub b-semiring $Q$ of $S$ is called a quasi ideal if it is $Q$ is generalized quasi ideal.

Lemma 4.4. The generalized 2-quasi ideal $Q$ is a 2-quasi ideal in $S$ if $Q$ is closed under ".".

Proof. Suppose that $Q$ is generalized 2-quasi ideal in $S$ which is closed under ".". Now, $Q+Q \subseteq Q+S$ and $Q+Q \subseteq S+Q$ implies $Q+Q \subseteq(Q+S) \cap(S+Q) \subseteq Q$. Thus, $Q$ is a 2-quasi ideal in $S$.

Remark 4.5. The generalized 2-quasi ideal fails to be a 2quasi ideal in $S$ by the Example 4.6.

Example 4.6. Consider the $b$-semiring $(S,+, \cdot)$ by the following table.

\begin{tabular}{|c|c|c|c|c|c|c|}
\hline+ & $s_{1}$ & $s_{2}$ & $s_{3}$ & $s_{4}$ & $s_{5}$ & $s_{6}$ \\
\hline$s_{1}$ & $s_{1}$ & $s_{1}$ & $s_{1}$ & $s_{1}$ & $s_{1}$ & $s_{1}$ \\
\hline$s_{2}$ & $s_{2}$ & $s_{2}$ & $s_{2}$ & $s_{2}$ & $s_{2}$ & $s_{2}$ \\
\hline$s_{3}$ & $s_{3}$ & $s_{3}$ & $s_{3}$ & $s_{3}$ & $s_{3}$ & $s_{3}$ \\
\hline$s_{4}$ & $s_{4}$ & $s_{4}$ & $s_{4}$ & $s_{4}$ & $s_{4}$ & $s_{4}$ \\
\hline$s_{5}$ & $s_{5}$ & $s_{5}$ & $s_{5}$ & $s_{5}$ & $s_{5}$ & $s_{5}$ \\
\hline$s_{6}$ & $s_{6}$ & $s_{6}$ & $s_{6}$ & $s_{6}$ & $s_{6}$ & $s_{6}$ \\
\hline
\end{tabular}

\begin{tabular}{|c|c|c|c|c|c|c|}
\hline$\cdot$ & $s_{1}$ & $s_{2}$ & $s_{3}$ & $s_{4}$ & $s_{5}$ & $s_{6}$ \\
\hline$s_{1}$ & $s_{1}$ & $s_{1}$ & $s_{1}$ & $s_{1}$ & $s_{1}$ & $s_{1}$ \\
\hline$s_{2}$ & $s_{1}$ & $s_{2}$ & $s_{2}$ & $s_{2}$ & $s_{2}$ & $s_{2}$ \\
\hline$s_{3}$ & $s_{1}$ & $s_{3}$ & $s_{3}$ & $s_{3}$ & $s_{3}$ & $s_{3}$ \\
\hline$s_{4}$ & $s_{1}$ & $s_{2}$ & $s_{2}$ & $s_{4}$ & $s_{4}$ & $s_{4}$ \\
\hline$s_{5}$ & $s_{1}$ & $s_{3}$ & $s_{3}$ & $s_{5}$ & $s_{5}$ & $s_{5}$ \\
\hline$s_{6}$ & $s_{1}$ & $s_{3}$ & $s_{3}$ & $s_{6}$ & $s_{6}$ & $s_{6}$ \\
\hline
\end{tabular}

Clearly, $\left\{s_{1}, s_{2}, s_{5}\right\}$ is a generalized 2-quasi ideal, but $s_{5} \cdot s_{2} \notin$ $\left\{s_{1}, s_{2}, s_{5}\right\}$ implies $\left\{s_{1}, s_{2}, s_{5}\right\}$ is not 2-quasi ideal in $S$.

Lemma 4.7. Every weak-2 right (left) ideal is a 2-quasi ideal in $S$.

Remark 4.8. Converse of the Lemma 4.7 fails by the Example 4.9.

Example 4.9. In Example 4.6, $\left\{s_{3}, s_{5}\right\}$ is a 2-quasi ideal, but $s_{1}+s_{5} \notin\left\{s_{3}, s_{5}\right\}$ implies $\left\{s_{3}, s_{5}\right\}$ not weak-2-left ideal in $S$.

Theorem 4.10. The intersection of weak-2 right ideal with weak-2 left ideal in $S$ is a 2-quasi ideal.

Proof. For the weak-2 right ideal $A$ and weak-2 left ideal $B$ in $S, A \cap B$ is a sub b-semiring. Now, $[(A \cap B)+S] \cap[S+(A \cap$ $B)] \subseteq(A+S) \cap(S+B) \subseteq A \cap B$ implies $A \cap B$ is a 2-quasi ideal in $S$.
Theorem 4.11. For any $a \in S$, the generalized 2-quasi ideal generated by " $a$ ", denoted by $\langle a\rangle_{g 2 q}$ is given by $\{a\} \cup[(a+$ $S) \cap(S+a)]$.

Proof. Now, $x \in(a+S) \cap(S+a)$, then $(x+S) \cap(S+x) \subseteq(a+$ $S) \cap(S+a)$. Thus $\{a\} \cup[(a+S) \cap(S+a)]$ is a generalized 2-quasi ideal in $S$. If $A$ is a generalized 2-quasi ideal in $S$ such that $a \in A$, then $\{a\} \cup[(a+S) \cap(S+a)] \subseteq A$. Therefore

$\langle a\rangle_{g 2 q}$ is the generalized 2-quasi ideal generated by " $a$ ".

Corollary 4.12. For a subset $A$ of $S, A \cup[(A+S) \cap(S+A)]$ is the generalized 2-quasi ideal generated by a set $A$ in $S$.

Theorem 4.13. For any $a \in S$, the 2-quasi ideal generated by " $a$ ", denoted by $<a>_{2 q}$ is given by $\left\{a^{m} \mid m \in \mathbb{Z}^{+}\right\} \cup[(a+$ $S) \cap(S+a)]$.

Proof. Clearly, $\left\{a^{m} \mid m \in \mathbb{Z}^{+}\right\} \cup[(a+S) \cap(S+a)]$ is generalized 2-quasi ideal. For $x, y \in[(a+S) \cap(S+a)], x \cdot y=a+\left(s_{1}\right.$. $\left.s_{3}\right) \in a+S$ and $x \cdot y=\left(s_{2} \cdot s_{4}\right)+a \in S+a$ imply $x \cdot y \in[(a+$ $S) \cap(S+a)]$. For $x \in\left\{a^{m} \mid m \in \mathbb{Z}^{+}\right\}, y \in[(a+S) \cap(S+a)]$ and by Lemma 3.12, $x \cdot y=a^{m} \cdot\left(a+s_{3}\right)=a+\left[\left(a^{m} \cdot s_{3}\right)^{m+1}\right] \in a+S$ and $x \cdot y=a^{m} \cdot\left(s_{4}+a\right)=\left[\left(a^{m} \cdot s_{4}\right)^{m+1}\right]+a \in S+a$. Thus, $x \cdot y \in[(a+S) \cap(S+a)]$. Similarly, $y \cdot x \in[(a+S) \cap(S+a)]$. By Lemma 4.4, $\left\{a^{m}\right\} \cup[(a+S) \cap(S+a)]$ is a 2-quasi ideal in $S$. If $A$ is a 2-quasi ideal in $S$ such that $a \in A$, then $\left\{a^{m}\right\} \cup[(a+S) \cap(S+a)] \subseteq A$. Hence $<a>_{2 q}$ is the 2-quasi ideal generated by " $a$ ".

Corollary 4.14. For a subset $A$ of $S, \Pi A \cup\left[\Pi(A+S) \cap \prod(S+\right.$ $A)]$ is the 2-quasi ideal generated by a set $A$ in $S$.

Theorem 4.15. For a b-semiring $S$, the following conditions are equivalent.

(1) $S$ is 2-regular.

(2) $R \cap Q_{1} \subseteq R+Q_{1}$, for the weak-2 right ideals $R$ and generalized 2-quasi ideals $Q_{1}$.

(3) $R \cap Q \subseteq R+Q$, for the weak-2 right ideals $R$ and 2-quasi ideals $Q$.

(4) $Q_{1} \cap L \subseteq Q_{1}+L$, for the generalized 2-quasi ideals $Q_{1}$ and weak-2 left ideals $L$.

(5) $Q \cap L \subseteq Q+L$, for the 2-quasi ideals $Q$ and weak-2 left ideals $L$.

(6) $R \cap L=R+L$, for the weak-2 right ideals $R$ and weak- 2 left ideals $L$.

Proof. First, we prove that $(1) \Rightarrow(2) \Rightarrow(3) \Rightarrow(6) \Rightarrow(1)$ and (1) $\Rightarrow(4) \Rightarrow(5) \Rightarrow(6)$.

(1) $\Rightarrow(2)$ For $a \in R \cap Q_{1}$, then there exist $s \in S$ such that $a=(a+s)+a$. Thus, $R \cap Q_{1} \subseteq R+Q_{1}$.

(2) $\Rightarrow$ (3) Straightforward.

(3) $\Rightarrow$ (6) By Lemma 4.7, $R \cap L \subseteq R+L$. Now, $R+L \subseteq$ $R+S \subseteq R$ and $R+L \subseteq S+L \subseteq L$. Then (6) follows.

(6) $\Rightarrow(1)$ The proof follows from Theorem 3.16.

(1) $\Rightarrow(4)$ For $a \in Q_{1} \cap L$, then there exist $s \in S$ such that $a=a+(s+a)$. Thus, $Q_{1} \cap L \subseteq Q_{1}+L$.

(4) $\Rightarrow(5)$ By Lemma 4.5, (5) holds.

$(5) \Rightarrow(6)$ The proof follows from Lemma 4.7. 
Theorem 4.16. For a b-semiring $S$, the following conditions are equivalent.

(1) $S$ is 2-regular.

(2) $Q_{1} \cap I \cap Q_{2} \subseteq Q_{1}+I+Q_{2}$, for the generalized 2-quasi ideals $Q_{1}$ and $Q_{2}$ and weak-2 ideals $I$.

(3) $Q_{1} \cap I \cap Q \subseteq Q_{1}+I+Q$, for the generalized 2-quasi ideals $Q_{1}$, weak-2 ideals I and 2-quasi ideals $Q$.

(4) $Q \cap I \cap Q_{2} \subseteq Q+I+Q_{2}$, for the 2-quasi ideals $Q$, weak-2 ideals I and generalized 2-quasi ideals $Q_{2}$.

(5) $Q \cap I \cap Q \subseteq Q+I+Q$, for the 2-quasi ideals $Q$ and weak-2 ideals $I$.

(6) $Q_{1} \cap I \cap L \subseteq Q_{1}+I+L$, for the generalized 2-quasi ideals $Q_{1}$, weak-2 ideals I and weak-2 left ideals $L$.

(7) $Q \cap I \cap L \subseteq Q+I+L$, for the 2-quasi ideals $Q$, weak-2 ideals I and weak-2 left ideals $L$.

(8) $R \cap I \cap Q_{2} \subseteq R+I+Q_{2}$, for the weak-2 right ideals $R$, weak-2 ideals I and generalized 2-quasi ideals $Q_{2}$.

(9) $R \cap I \cap Q \subseteq R+I+Q$, for the weak-2 right ideals $R$, weak2 ideals I and 2-quasi ideals $Q$.

(10) $R \cap I \cap L \subseteq R+I+L$, for the weak-2 right ideals $R$, weak2 ideals I and weak-2 left ideals $L$.

(11) $R \cap L=R+L$, for the weak-2 right ideals $R$ and weak-2 left ideals $L$.

(12) $Q_{1} \cap I \subseteq Q_{1}+I+Q_{1}$, for the generalized 2-quasi ideals $Q_{1}$ and weak-2 ideals $I$.

(13) $Q \cap I \subseteq Q+I+Q$, for the 2-quasi ideals $Q$ and weak-2 ideals $I$.

(14) $Q_{1}=Q_{1}+S+Q_{1}$, for the generalized 2-quasi ideals $Q_{1}$.

(15) $Q=Q+S+Q$, for the 2-quasi ideals $Q$.

Proof. First, we prove that (1) $\Rightarrow(2) \Rightarrow(3) \Rightarrow(5) \Rightarrow(11) \Rightarrow$ (1), (3) $\Rightarrow(6) \Rightarrow(7) \Rightarrow(11),(2) \Rightarrow(4) \Rightarrow(8) \Rightarrow(9) \Rightarrow$ $(10) \Rightarrow(11),(12) \Rightarrow(14) \Rightarrow(1)$ and $(2) \Rightarrow(12) \Rightarrow(13) \Rightarrow$ $(15) \Rightarrow(1)$.

(1) $\Rightarrow(2)$ For $a \in Q_{1} \cap I \cap Q_{2}$, then there exists $s \in S$ such that $a=a+s+a$. Thus, $a=a+(s+a+s)+a \in Q_{1}+I+Q_{2}$.

Then (2) follows.

(2) $\Rightarrow$ (3) Straightforward.

(3) $\Rightarrow(5)$ Straightforward.

(5) $\Rightarrow(11)$ By taking $I=S$ in (5), $R \cap L \subseteq R+L$. Thus, $R \cap L=R+L$.

$(11) \Rightarrow(1)$ The proof follows from Theorem 3.16.

$(3) \Rightarrow(6)$ The proof follows from Lemma 4.7.

(6) $\Rightarrow$ (7) Straightforward.

(7) $\Rightarrow$ (11) By taking $I=S, R \cap L \subseteq R+L$. Thus, $R \cap L=$ $R+L$.

(2) $\Rightarrow$ (4) Straightforward.

(4) $\Rightarrow$ (8) By Lemma 4.7, the result holds.

(8) $\Rightarrow(9)$ Straightforward.

(9) $\Rightarrow(10)$ By Lemma 4.7, (10) holds.

(10) $\Rightarrow(11)$ Taking $I=S$ in (10), $R \cap L \subseteq R+L$. Thus, $R \cap$ $L=R+L$.

$(12) \Rightarrow(14)$ By (12), $Q_{1} \subseteq Q_{1}+S+Q_{1} \subseteq\left[\left(Q_{1}+S\right) \cap(S+\right.$ $\left.\left.Q_{1}\right)\right] \subseteq Q_{1}$. Thus, (14) holds .

(14) $\Rightarrow(1)$ For any $a \in S, a \in<a>_{g 2 q}+S+\left\langle a>_{g 2 q}\right.$ and by Theorem 4.11, $a \in[a+S+a] \cup[a+S+[(a+S) \cap(S+$ $a)]] \cup[[(a+S) \cap(S+a)]+S+a] \cup[[(a+S) \cap(S+a)]+S+$
$[(a+S) \cap(S+a)]]$. Thus, $a \in a+S+a$. Therefore $S$ is 2 regular.

(2) $\Rightarrow$ (12) Taking $Q_{2}=Q_{1}$ in (2), we get the result.

$(12) \Rightarrow$ (13) Straightforward.

$(13) \Rightarrow(15)$ By (13), $Q \subseteq Q+S+Q \subseteq[(Q+S) \cap(S+Q)] \subseteq$ $Q$ implies $Q=Q+S+Q$.

$(15) \Rightarrow(1)$ For any $a \in S$ by (15), $\left.a \in<a\rangle_{2 q}+S+<a\right\rangle_{2 q}$ and by Theorem 4.13 and Lemma 3.12, $a \in\left[a^{n}+S+a^{m}\right] \cup$ $\left[a^{n}+S+[(a+S) \cap(S+a)]\right] \cup\left[[(a+S) \cap(S+a)]+S+a^{m}\right] \cup$ $[[(a+S) \cap(S+a)]+S+[(a+S) \cap(S+a)]]$. Thus, $a \in a+$ $S+a$. Hence $S$ is 2-regular.

Theorem 4.17. For a $b$-semiring $S$, the following conditions are equivalent.

(1) $S$ is regular.

(2) $R \cap Q_{1} \subseteq\left(R \cdot Q_{1}\right) \cap\left(R+Q_{1}\right)$, for the right ideals $R$ and generalized quasi ideals $Q_{1}$.

(3) $R \cap Q \subseteq(R \cdot Q) \cap(R+Q)$, for the right ideals $R$ and quasi ideals $Q$.

(4) $Q_{1} \cap L \subseteq\left(Q_{1} \cdot L\right) \cap\left(Q_{1}+L\right)$, for the generalized quasi ideals $Q_{1}$ and left ideals $L$.

(5) $Q \cap L \subseteq(Q \cdot L) \cap(Q+L)$, for the quasi ideals $Q$ and left ideals $L$.

(6) $R \cap L=(R \cdot L) \cap(R+L)$, for the right ideals $R$ and left ideals $L$.

Proof. First, we prove that $(1) \Rightarrow(2) \Rightarrow(3) \Rightarrow(6) \Rightarrow(1)$ and $(1) \Rightarrow(4) \Rightarrow(5) \Rightarrow(6)$.

$(1) \Rightarrow(2)$ By Theorem 3.17, $R \cap Q_{1} \subseteq R \cdot Q_{1}$ and by Theorem 4.15, $R \cap Q_{1} \subseteq R+Q_{1}$. Thus, $R \cap Q_{1} \subseteq\left(R \cdot Q_{1}\right) \cap\left(R+Q_{1}\right)$

(2) $\Rightarrow$ (3) The proof follows from Theorem 3.17 and 4.15.

(3) $\Rightarrow(6)$ By Theorem 3.17, $R \cap L=R \cdot L$ and by Theorem $4.15, R \cap L=R+L$. Then (6) follows.

(6) $\Rightarrow(1)$ Now, $R \cdot L \subseteq R \cap L=(R \cdot L) \cap(R+L) \subseteq R \cdot L$, then by Theorem 3.16, $S$ is 1-regular. Similarly, $R+L \subseteq R \cap L=$ $(R \cdot L) \cap(R+L) \subseteq R+L$. Then by Theorem 3.16, $S$ is 2 regular. Thus, $S$ is regular.

$(1) \Rightarrow(4)$ By Theorem 3.17 and 4.15, $Q_{1} \cap L \subseteq Q_{1} \cdot L$ and $Q_{1} \cap L \subseteq Q_{1}+L$. Then (4) follows.

$(4) \Rightarrow(5)$ The proof follows from Theorem 3.17 and 4.15.

$(5) \Rightarrow(6)$ By Lemma 3.6 and 4.7, (6) holds.

Theorem 4.18. For a $b$-semiring $S$, the following conditions are equivalent.

(1) $S$ is regular.

(2) $Q_{1} \cap I \cap Q_{2} \subseteq\left(Q_{1} \cdot I \cdot Q_{2}\right) \cap\left(Q_{1}+I+Q_{2}\right)$, for the generalized quasi ideals $Q_{1}$ and $Q_{2}$ and ideals $I$.

(3) $Q_{1} \cap I \cap Q \subseteq\left(Q_{1} \cdot I \cdot Q\right) \cap\left(Q_{1}+I+Q\right)$, for the generalized quasi ideals $Q_{1}$, ideals I and quasi ideals $Q$.

(4) $Q \cap I \cap Q_{2} \subseteq\left(Q \cdot I \cdot Q_{2}\right) \cap\left(Q+I+Q_{2}\right)$, for the quasi ideals $Q$, ideals I and generalized quasi ideals $Q_{2}$.

(5) $Q \cap I \cap Q \subseteq(Q \cdot I \cdot Q) \cap(Q+I+Q)$, for the quasi ideals $Q$ and ideals $I$.

(6) $Q_{1} \cap I \cap L \subseteq\left(Q_{1} \cdot I \cdot L\right) \cap\left(Q_{1}+I+L\right)$, for the generalized quasi ideals $Q_{1}$, ideals $I$ and left ideals $L$.

(7) $Q \cap I \cap L \subseteq(Q \cdot I \cdot L) \cap(Q+I+L)$, for the quasi ideals $Q$, ideals I and left ideals $L$. 
(8) $R \cap I \cap Q_{2} \subseteq\left(R \cdot I \cdot Q_{2}\right) \cap\left(R+I+Q_{2}\right)$, for the right ideals $R$, ideals $I$ and generalized quasi ideals $Q_{2}$.

(9) $R \cap I \cap Q \subseteq(R \cdot I \cdot Q) \cap(R+I+Q)$, for the right ideals $R$, ideals $I$ and quasi ideals $Q$.

(10) $R \cap I \cap L \subseteq(R \cdot I \cdot L) \cap(R+I+L)$, for the right ideals $R$, ideals $I$ and left ideals $L$.

(11) $R \cap L=(R \cdot L) \cap(R+L)$, for the right ideals $R$ and left ideals $L$.

(12) $Q_{1} \cap I \subseteq\left(Q_{1} \cdot I \cdot Q_{1}\right) \cap\left(Q_{1}+I+Q_{1}\right)$, for the generalized quasi ideals $Q_{1}$ and ideals $I$.

(13) $Q \cap I \subseteq(Q \cdot I \cdot Q) \cap(Q+I+Q)$, for the quasi ideals $Q$ and ideals $I$.

(14) $Q_{1}=\left(Q_{1} \cdot S \cdot Q_{1}\right) \cap\left(Q_{1}+S+Q_{1}\right)$, for the generalized quasi ideals $Q_{1}$.

(15) $Q=(Q \cdot S \cdot Q) \cap(Q+S+Q)$, for the quasi ideals $Q$.

Proof. First, we prove that $(1) \Rightarrow(2) \Rightarrow(3) \Rightarrow(5) \Rightarrow(11) \Rightarrow$ (1), (3) $\Rightarrow(6) \Rightarrow(7) \Rightarrow(11),(2) \Rightarrow(4) \Rightarrow(8) \Rightarrow(9) \Rightarrow$ $(10) \Rightarrow(11),(12) \Rightarrow(14) \Rightarrow(1)$ and $(2) \Rightarrow(12) \Rightarrow(13) \Rightarrow$ $(15) \Rightarrow(1)$.

$(1) \Rightarrow(2)$ By Theorem 3.18, $Q_{1} \cap I \cap Q_{2} \subseteq\left(Q_{1} \cdot I \cdot Q_{2}\right)$ and by Theorem 4.16, $Q_{1} \cap I \cap Q_{2} \subseteq\left(Q_{1}+I+Q_{2}\right)$. Thus, (2)holds.

(2) $\Rightarrow(3)$ By Theorem 3.18 and 4.16, (3) holds.

(3) $\Rightarrow(5)$ The proof follows from Lemma 3.6 and 4.7.

(5) $\Rightarrow$ (11) By Theorem 3.18 and 4.16, (11) holds.

$(11) \Rightarrow$ (1) The proof follows from Theorem 4.17 .

$(3) \Rightarrow(6)$ The proof follows from Theorem 3.18 and 4.16 .

$(6) \Rightarrow(7)$ By Theorem 3.18 and 4.16, (7) holds.

(7) $\Rightarrow$ (11) By Theorem $3.18, R \cap L=R \cdot L$ and by Theorem

4.16, $R \cap L=R+L$. Thus, (11) holds.

$(2) \Rightarrow(4)$ By Theorem 3.18 and 4.16, (4) holds.

$(4) \Rightarrow(8)$ The proof follows from Lemma 3.6 and 4.7.

$(8) \Rightarrow(9)$ The proof follows from Theorem 3.18 and 4.16 .

$(9) \Rightarrow(10)$ By Lemma 3.6 and Lemma 4.7, (10) holds.

$(10) \Rightarrow(11)$ The proof follows from Theorem 3.18 and 4.16.

$(12) \Rightarrow(14)$ By Theorem 3.18 and 4.16, $Q_{1}=Q_{1} \cdot S \cdot Q_{1}$ and $Q_{1}=Q_{1}+S+Q_{1}$. Thus, (14)holds.

(14) $\Rightarrow$ (1) Now, $Q_{1} \subseteq Q_{1} \cdot S \cdot Q_{1} \subseteq\left[\left(Q_{1} \cdot S\right) \cap\left(S \cdot Q_{1}\right)\right] \subseteq Q_{1}$. Then $Q_{1}=Q_{1} \cdot S \cdot Q_{1}$. By Theorem 3.18, $S$ is 1-regular. Then, $Q_{1} \subseteq Q_{1}+S+Q_{1} \subseteq\left[\left(Q_{1}+S\right) \cap\left(S+Q_{1}\right)\right] \subseteq Q_{1}$. Thus $Q_{1}=$ $Q_{1}+S+Q_{1}$. By Theorem 4.16, $S$ is 2-regular. Thus, $S$ is regular.

(2) $\Rightarrow(12)$ Taking $Q_{2}=Q_{1}$ in (2), we get the result.

$(12) \Rightarrow(13)$ By Theorem 3.18 and 4.16, (13) holds.

$(13) \Rightarrow(15)$ By Theorem 3.18 and 4.16, $Q=Q \cdot S \cdot Q$ and $Q=Q+S+Q$. Thus, (15)holds.

$(15) \Rightarrow(1)$ Now, $Q \subseteq Q \cdot S \cdot Q \subseteq[(Q \cdot S) \cap(S \cdot Q)] \subseteq Q$. Then $Q=Q \cdot S \cdot Q$. By Theorem 3.18, $S$ is 1-regular. Then, $Q \subseteq$ $Q+S+Q \subseteq[(Q+S) \cap(S+Q)] \subseteq Q$. Thus $Q=Q+S+Q$. By Theorem 4.16, $S$ is 2-regular. Thus, $S$ is regular.

Theorem 4.19. For any $a \in S$, the generalized quasi ideal generated by " $a$ ", denoted by $\langle a\rangle_{g q}$ is given by $\{a\} \cup[(a$. $S) \cap(S \cdot a)] \cup[(a+S) \cap(S+a)] \cup[[(a \cdot S)+S] \cap[S+(S \cdot a)]]$.

Proof. By Theorem 3.10 and 4.11, $\{a\} \cup[(a \cdot S) \cap(S \cdot a)]$ and $\{a\} \cup[(a+S) \cap(S+a)]$ is a generalized 1-quasi ideal and generalized 2-quasi ideal of $S$ respectively.

For $x \in[(a \cdot S) \cap(S \cdot a)], x+s^{\prime}=\left(a \cdot s_{1}\right)+s^{\prime} \in[(a \cdot S)+S]$ and $s^{\prime \prime}+x=s^{\prime \prime}+\left(s_{2} \cdot a\right) \in[S+(S \cdot a)]$ imply $[[(a \cdot S) \cap(S \cdot a)]+$ $S] \cap[S+[(a \cdot S) \cap(S \cdot a)]] \subseteq[[(a \cdot S)+S] \cap[S+(S \cdot a)]]$.

For $x \in[(a+S) \cap(S+a)], x \cdot s^{\prime}=\left(a+s_{1}\right) \cdot s^{\prime} \in[(a \cdot S)+S]$ and $s^{\prime \prime} \cdot x=s^{\prime \prime} \cdot\left(s_{2}+a\right) \in[S+(S \cdot a)]$ imply $[[(a+S) \cap(S+a)] \cdot S]$ $\cap[S \cdot[(a+S) \cap(S+a)]] \subseteq[[(a \cdot S)+S] \cap[S+(S \cdot a)]]$.

Now, $[(a \cdot S)+S]+S \subseteq[(a \cdot S)+S]$ and $S+[S+(S \cdot a)] \subseteq$ $[S+(S \cdot a)]$ imply $[[[(a \cdot S)+S] \cap[S+(S \cdot a)]]+S] \cap[S+[[(a \cdot$ $S)+S] \cap[S+(S \cdot a)]]] \subseteq[[(a \cdot S)+S] \cap[S+(S \cdot a)]]$. Now, $[(a \cdot S)+S] \cdot S \subseteq[(a \cdot S)+S]$ and $S \cdot[S+(S \cdot a)] \subseteq[S+(S \cdot a)]$ imply $[[[(a \cdot S)+S] \cap[S+(S \cdot a)]] \cdot S] \cap[S \cdot[[(a \cdot S)+S] \cap$ $[S+(S \cdot a)]]] \subseteq[[(a \cdot S)+S] \cap[S+(S \cdot a)]]$.

Thus, $\{a\} \cup[(a \cdot S) \cap(S \cdot a)] \cup[(a+S) \cap(S+a)] \cup[[(a \cdot S)+$ $S] \cap[S+(S \cdot a)]]$ is a generalized quasi ideal in $S$. If $A$ is a generalized quasi ideal in $S$ such that $a \in A$, then $\{a\} \cup[(a$. $S) \cap(S \cdot a)] \cup[(a+S) \cap(S+a)] \cup[[(a \cdot S)+S] \cap[S+(S \cdot a)]] \subseteq$ $A$. Thus, $\langle a\rangle_{g q}$ is a generalized quasi ideal generated by " $a$ ".

Theorem 4.20. For any $a \in S$, the quasi ideal generated by " $a$ ", denoted by $\langle a\rangle_{q}$ is given by $\left\{n a \mid n \in \mathbb{Z}^{+}\right\} \cup\left\{a^{m} \mid m \in\right.$ $\left.\mathbb{Z}^{+}\right\} \cup[(a \cdot S) \cap(S \cdot a)] \cup[(a+S) \cap(S+a)] \cup[[(a \cdot S)+S] \cap$ $[S+(S \cdot a)]]$.

Proof. By Theorem 4.19, $\{a\} \cup[(a \cdot S) \cap(S \cdot a)] \cup[(a+S) \cap$ $(S+a)] \cup[[(a \cdot S)+S] \cap[S+(S \cdot a)]]$ is a generalized quasi ideal of $S$. Now,

$\left[\left(\left\{n a \mid n \in \mathbb{Z}^{+}\right\}+S\right) \cap\left(S+\left\{n a \mid n \in \mathbb{Z}^{+}\right\}\right)\right] \subseteq[(a+S) \cap(S+$ $a)]$ and $\left[\left(\left\{a^{m} \mid m \in \mathbb{Z}^{+}\right\} \cdot S\right) \cap\left(S \cdot\left\{a^{m} \mid m \in \mathbb{Z}^{+}\right\}\right)\right] \subseteq[(a \cdot S) \cap$ $(S \cdot a)]$.

By Theorem 3.13 and 4.13, $Q=\left\{n a \mid n \in \mathbb{Z}^{+}\right\} \cup\left\{a^{m} \mid m \in\right.$ $\left.\mathbb{Z}^{+}\right\} \cup[(a \cdot S) \cap(S \cdot a)] \cup[(a+S) \cap(S+a)] \cup[[(a \cdot S)+S] \cap$ $[S+(S \cdot a)]],\left\{n a \mid n \in \mathbb{Z}^{+}\right\} \cup[(a \cdot S) \cap(S \cdot a)]$ and $\left\{a^{m} \mid m \in\right.$ $\left.\mathbb{Z}^{+}\right\} \cup[(a+S) \cap(S+a)]$ are generalized quasi ideal and sub $b$-semirings of $S$ respectively.

Now, $\left\{n a \mid n \in \mathbb{Z}^{+}\right\}+\left\{a^{m} \mid m \in \mathbb{Z}^{+}\right\} \subseteq[(a+S) \cap(S+a)] \subseteq Q$ and $\left\{a^{m} \mid m \in \mathbb{Z}^{+}\right\}+\left\{n a \mid n \in \mathbb{Z}^{+}\right\} \subseteq[(a+S) \cap(S+a)] \subseteq Q$ and $\left\{n a \mid n \in \mathbb{Z}^{+}\right\} \cdot\left\{a^{m} \mid m \in \mathbb{Z}^{+}\right\} \subseteq[(a \cdot S) \cap(S \cdot a)] \subseteq Q$ and $\left\{a^{m} \mid m \in \mathbb{Z}^{+}\right\} \cdot\left\{n a \mid n \in \mathbb{Z}^{+}\right\} \subseteq[(a \cdot S) \cap(S \cdot a)] \subseteq Q$.

Let $x \in\left\{n a \mid n \in \mathbb{Z}^{+}\right\}$and $y \in[(a+S) \cap(S+a)]$. Then $x+y=$ $n a+\left(a+s_{1}\right)=(n+1) a+s_{1}=a+\left(n a+s_{1}\right) \in a+S$ and $x+y=n a+\left(s_{2}+a\right)=\left(n a+s_{2}\right)+a \in S+a$ imply $\{n a \mid n \in$ $\left.\mathbb{Z}^{+}\right\}+[(a+S) \cap(S+a)] \subseteq[(a+S) \cap(S+a)] \subseteq Q$. Similarly, $[(a+S) \cap(S+a)]+\left\{n a \mid n \in \mathbb{Z}^{+}\right\} \subseteq[(a+S) \cap(S+a)] \subseteq Q$.

Now, $x \cdot y=n a \cdot\left(a+s_{1}\right)=\left[a \cdot\left(a+s_{1}\right)\right]+\left[(n-1) a \cdot\left(a+s_{1}\right)\right] \in$ $[(a \cdot S)+S]$ and $x \cdot y=n a \cdot\left(s_{2}+a\right)=\left(n a \cdot s_{2}\right)+(n a \cdot a) \in$ $[S+(S \cdot a)]$ imply $\left\{n a \mid n \in \mathbb{Z}^{+}\right\} \cdot[(a+S) \cap(S+a)] \subseteq[[(a \cdot$ 
$S)+S] \cap[S+(S \cdot a)]] \subseteq Q$ and $[(a+S) \cap(S+a)] \cdot\{n a \mid n \in$ $\left.\mathbb{Z}^{+}\right\} \subseteq[[(a \cdot S)+S] \cap[S+(S \cdot a)]] \subseteq Q$.

Let $x \in\left\{n a \mid n \in \mathbb{Z}^{+}\right\}$and $y \in[[(a \cdot S)+S] \cap[S+(S \cdot a)]]$. Then $x+y=n a+\left[\left(a \cdot s_{1}\right)+s_{2}\right]=\left[(n+1) a \cdot\left(n a+s_{1}\right)\right]+s_{2} \in$ $[(a \cdot S)+S]$ and $x+y=\left(n a+s_{3}\right)+\left(s_{4} \cdot a\right) \in[S+(S \cdot a)]$ imply $x+y \in[[(a \cdot S)+S] \cap[S+(S \cdot a)]] \subseteq Q$. Now, $x \cdot y=$ $n a \cdot\left[\left(a \cdot s_{1}\right)+s_{2}\right]=\left[\left(a \cdot n\left(a \cdot s_{1}\right)\right)+\left(n a \cdot s_{2}\right)\right] \in[(a \cdot S)+S]$ and $x \cdot y=n a \cdot\left[s_{3}+\left(s_{4} \cdot a\right)\right]=\left[\left(n a \cdot s_{3}\right)+\left(\left(n a \cdot s_{4}\right) \cdot a\right)\right] \in$ $[S+(S \cdot a)]$ imply $x \cdot y \in[[(a \cdot S)+S] \cap[S+(S \cdot a)]] \subseteq Q$.

Similarly, $y+x$ and $y \cdot x \in[[(a \cdot S)+S] \cap[S+(S \cdot a)]] \subseteq Q$. Let $x \in\left\{a^{m} \mid m \in \mathbb{Z}^{+}\right\}$and $y \in[(a \cdot S) \cap(S \cdot a)]$. Then , $x+y=$ $a^{m}+\left(a \cdot s_{1}\right)=\left(a \cdot a^{m-1}\right)+\left(a \cdot s_{1}\right)=\left[a+\left(a \cdot s_{1}\right)\right] \cdot\left[a^{m-1}+\right.$ $\left.\left(a \cdot s_{1}\right)\right] \in[(a \cdot S)+S]$ and $x+y=a^{m}+\left(s_{3} \cdot a\right)=\left(a^{m}+s_{3}\right)$. $\left(a^{m}+a\right)=\left[\left(a^{m}+s_{3}\right) \cdot a^{m}\right]+\left[\left(a^{m}+s_{3}\right) \cdot a\right] \in[S+(S \cdot a)]$ imply $x+y \in[[(a \cdot S)+S] \cap[S+(S \cdot a)]] \subseteq Q$.

Now,$x \cdot y=a^{m} \cdot\left(a \cdot s_{1}\right) \in a \cdot S$ and $x \cdot y=a^{m} \cdot\left(s_{2} \cdot a\right)=\left(a^{m} \cdot s_{2}\right)$. $a \in S \cdot a$ imply $x \cdot y \in[(a \cdot S) \cap(S \cdot a)] \subseteq Q$. Similarly, $y+x \in$ $[[(a \cdot S)+S] \cap[S+(S \cdot a)]] \subseteq Q$ and $y \cdot x \in[(a \cdot S) \cap(S \cdot a)] \subseteq Q$. Let $x \in\left\{a^{m} \mid m \in \mathbb{Z}^{+}\right\}$and $y \in[[(a \cdot S)+S] \cap[S+(S \cdot a)]]$. Now, $x+y=a^{m}+\left[\left(a \cdot s_{1}\right)+s_{2}\right]=\left(a \cdot a^{m-1}\right)+\left[\left(a \cdot s_{1}\right)+s_{2}\right]=$ $\left[a+\left[\left(a \cdot s_{1}\right)+s_{2}\right]\right] \cdot\left[a^{m-1}+\left[\left(a \cdot s_{1}\right)+s_{2}\right]\right] \in[(a \cdot S)+S]$ and $\left.x+y=\left(a^{m}+s_{4}\right)+\left(s_{5} \cdot a\right)\right] \in[S+(S \cdot a)]$ imply $x+y \in[[(a$. $S)+S] \cap[S+(S \cdot a)]] \subseteq Q$. Now, $x \cdot y=a^{m} \cdot\left[\left(a \cdot s_{1}\right)+s_{2}\right]=$ $a \cdot\left[a^{m-1} \cdot\left(a \cdot s_{1}\right)\right]+\left(a^{m} \cdot s_{2}\right) \in[(a \cdot S)+S]$ and $x \cdot y=a^{m}$. $\left.\left[s_{4}+\left(s_{5} \cdot a\right)\right]=\left(a^{m} \cdot s_{4}\right)+\left[\left(a^{m} \cdot s_{5}\right) \cdot a\right)\right] \in[S+(S \cdot a)]$ imply $x \cdot y \in[[(a \cdot S)+S] \cap[S+(S \cdot a)]] \subseteq Q$. Similarly, $y+x$ and $y \cdot x \in[[(a \cdot S)+S] \cap[S+(S \cdot a)]] \subseteq Q$.

Now, $(a \cdot S)+(a+S) \subseteq(a \cdot S)+S$ and $(S \cdot a)+(S+a)=$ $[S+(S+a)] \cdot[(a+S)+a] \subseteq S \cdot(S+a)$ imply $[[(a \cdot S) \cap(S$. $a)]+[(a+S) \cap(S+a)]] \subseteq[[(a \cdot S)+S] \cap[S+(S \cdot a)]] \subseteq Q$ and $[[(a+S) \cap(S+a)]+[(a \cdot S) \cap(S \cdot a)]] \subseteq[[(a \cdot S)+S] \cap$ $[S+(S \cdot a)]] \subseteq Q$

Now, $(a \cdot S) \cdot(a+S)=[(a \cdot S) \cdot a]+[(a \cdot S) \cdot S] \subseteq(a \cdot S)+S$ and $(S \cdot a) \cdot(S+a)=[(S \cdot a) \cdot S]+[(S \cdot a) \cdot a] \subseteq S \cdot(S+a)$ imply $[[(a \cdot S) \cap(S \cdot a)] \cdot[(a+S) \cap(S+a)]] \subseteq[[(a \cdot S)+S] \cap$ $[S+(S \cdot a)]] \subseteq Q$ and $[[(a+S) \cap(S+a)] \cdot[(a \cdot S) \cap(S \cdot a)]] \subseteq$ $[[(a \cdot S)+S] \cap[S+(S \cdot a)]] \subseteq Q$.

Now, $(a \cdot S)+[(a \cdot S)+S] \subseteq[(a \cdot S)+S]$ and $(S \cdot a)+[S+(S$. $a)] \subseteq[S+(S \cdot a)]$ imply $[(a \cdot S) \cap(S \cdot a)]+[[(a \cdot S)+S] \cap[S+$ $(S \cdot a)]] \subseteq[[(a \cdot S)+S] \cap[S+(S \cdot a)]] \subseteq Q$. Similarly, $[[(a \cdot$ $S)+S] \cap[S+(S \cdot a)]]+[(a \cdot S) \cap(S \cdot a)] \subseteq[[(a \cdot S)+S] \cap[S+$
$(S \cdot a)]] \subseteq Q$

Now, $(a \cdot S) \cdot[(a \cdot S)+S] \subseteq[(a \cdot S)+S]$ and $(S \cdot a) \cdot[S+(S \cdot a)]=$ $[(S \cdot a) \cdot S]+[(S \cdot a) \cdot(S \cdot a)] \subseteq[S+(S \cdot a)]$ imply $[(a \cdot S) \cap(S$. $a)] \cdot[[(a \cdot S)+S] \cap[S+(S \cdot a)]] \subseteq[[(a \cdot S)+S] \cap[S+(S \cdot$ $a)]] \subseteq Q$. Similarly, $[[(a \cdot S)+S] \cap[S+(S \cdot a)]] \cdot[(a \cdot S) \cap(S$. a) $] \subseteq[[(a \cdot S)+S] \cap[S+(S \cdot a)]] \subseteq Q$.

Now, $(a+S)+[(a \cdot S)+S] \subseteq(a+S)+[(a+S) \cdot S]=[(a+$ $S)+(a+S)] \cdot[(a+S)+S] \subseteq(a+S) \cdot S=[(a \cdot S)+S]$ and $(S+a)+[S+(S \cdot a)] \subseteq[S+(S \cdot a)]$ imply $[(a+S) \cap(S+a)]+$ $[[(a \cdot S)+S] \cap[S+(S \cdot a)]] \subseteq[[(a \cdot S)+S] \cap[S+(S \cdot a)]] \subseteq Q$. Similarly, $[[(a \cdot S)+S] \cap[S+(S \cdot a)]]+[(a+S) \cap(S+a)] \subseteq$ $[[(a \cdot S)+S] \cap[S+(S \cdot a)]] \subseteq Q$. Now, $(a+S) \cdot[(a \cdot S)+S] \subseteq$ $[(a+S) \cdot S]=[(a \cdot S)+S]$ and $(S+a) \cdot[S+(S \cdot a)] \subseteq[(S+a)$. $S]+[(S+a) \cdot(S \cdot a)] \subseteq[S+(S \cdot a)]$ imply $[(a+S) \cap(S+a)]$. $[[(a \cdot S)+S] \cap[S+(S \cdot a)]] \subseteq[[(a \cdot S)+S] \cap[S+(S \cdot a)]] \subseteq Q$. Similarly, $[[(a \cdot S)+S] \cap[S+(S \cdot a)]] \cdot[(a+S) \cap(S+a)] \subseteq$ $[[(a \cdot S)+S] \cap[S+(S \cdot a)]] \subseteq Q$.

Now, $[(a \cdot S)+S]+[(a \cdot S)+S] \subseteq[(a \cdot S)+S]$ and $[S+(S \cdot a)]+$ $[S+(S \cdot a)] \subseteq[S+(S \cdot a)]$ imply $[[(a \cdot S)+S] \cap[S+(S \cdot a)]]+$ $[[(a \cdot S)+S] \cap[S+(S \cdot a)]] \subseteq[[(a \cdot S)+S] \cap[S+(S \cdot a)]] \subseteq Q$. Now, $[(a \cdot S)+S] \cdot[(a \cdot S)+S] \subseteq[(a \cdot S)+S] \cdot S \subseteq[(a \cdot S)+S]$ and $[S+(S \cdot a)] \cdot[S+(S \cdot a)] \subseteq S \cdot[S+(S \cdot a)] \subseteq[S+(S \cdot a)]$ imply $[[(a \cdot S)+S] \cap[S+(S \cdot a)]] \cdot[[(a \cdot S)+S] \cap[S+(S$. $a)]] \subseteq[[(a \cdot S)+S] \cap[S+(S \cdot a)]] \subseteq Q$. Thus, $Q$ is a quasi ideal in $S$. If $A$ is a quasi ideal in $S$ such that $a \in A$, then $\left\{n a \mid n \in \mathbb{Z}^{+}\right\} \cup\left\{a^{m} \mid m \in \mathbb{Z}^{+}\right\} \cup[(a \cdot S) \cap(S \cdot a)] \cup[(a+S) \cap$ $(S+a)] \cup[[(a \cdot S)+S] \cap[S+(S \cdot a)]] \subseteq A$. Thus, $\langle a\rangle_{q}$ is the quasi ideal generated by " $a$ ".

\section{Acknowledgment}

The research of the second author is partially supported by "UGC-BSR grant : F.25-1/2014-15(BSR)/7 -254/2009(BSR) dated 20-01-2015" in India.

\section{References}

[1] G. Mohanraj, M.Palanikumar, Characterization of regular b-semirings, Mathematical Sciences International Research Journal, 7(2018), 117-123.

[2] G.Mohanraj, M.Palanikumar, Characterization of Various $\mathrm{k}$-Regular in b-Semirings, AIP Conference Proceedings, 2112(2019), 020021-1-020021-6.

[3] G.Mohanraj, M.Palanikumar, On various classes of one sided ideals in b-semirings, Submitted. 
[4] Ronnason Chinram, An introduction to b-semirings, Int. J. Contemp. Math. Science, 4(13)(2009), 649-657.

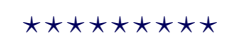

ISSN(P):2319-3786

Malaya Journal of Matematik

$\operatorname{ISSN}(\mathrm{O}): 2321-5666$

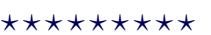

